Itinéraires Itinéraires

Littérature, textes, cultures

2011-1| 2011

Les Mémoires, une question de genre?

\title{
Le sexe des Mémoires
}

Jean-Louis Jeannelle

\section{(2) OpenEdition}

Journals

Édition électronique

URL : http://journals.openedition.org/itineraires/1596

DOI : $10.4000 /$ itineraires. 1596

ISSN : 2427-920X

Éditeur

Pléiade

Édition imprimée

Date de publication : 1 avril 2011

Pagination : 13-28

ISBN : 978-2-296-13692-2

ISSN : 2100-1340

Référence électronique

Jean-Louis Jeannelle, «Le sexe des Mémoires », Itinéraires [En ligne], 2011-1 | 2011, mis en ligne le 01 avril 2011, consulté le 25 juin 2020. URL : http://journals.openedition.org/itineraires/1596 ; DOI https://doi.org/10.4000/itineraires. 1596

\section{$\Theta \oplus \Theta \Theta$}

Itinéraires est mis à disposition selon les termes de la licence Creative Commons Attribution - Pas d'Utilisation Commerciale - Pas de Modification 4.0 International. 


\title{
Le sexe des Mémoires
}

\begin{abstract}
Everybody knows that since the beginning, women wrote memoirs. Why did so few women produce "egohistorical" narratives precisely from the moment they were granted a wide array of rights and achieved formal equality? Most of the time, the apparent disappearance of women writing memoirs during the twentieth century is explained by a natural evolution of the genres towards intimate forms of narratives. I would like to examine here if such a restriction - whether or not it is intentional - to the autobiographical model as opposed to those of "Souvenirs," memoirs, and other historical testimonies has diminished the place women should hold in field of self narratives, consequently confined them to a gynaeceum of life writings.
\end{abstract}

Keywords : memoirs, feminism, bibliography, Aragon, Proust

Mots clés : Mémoires, féminisme, bibliographie, Aragon, Proust

Dans la préface au premier tome de son Euvre poétique, paru aux éditions du Livre Club Diderot en 1974, Aragon avoue son trouble « à l'heure où se met en branle la confection (seul mot qui se propose à [lui], assez malencontreusement, pour désigner ce travail, fort étranger pourtant au travail de la couture) des Euvres poétiques complètes de quelqu'un qui porte [son] nom ${ }^{1} »$. Un astérisque renvoyant à une note en bas de page corrige aussitôt le titre donné à cette vaste entreprise éditoriale, qui fait suite à la publication, entre 1964 et 1974, de ses Euvres romanesques croisées à celles d'Elsa Triolet aux éditions Robert Laffont :

Je sais qu'on a préféré le titre au singulier: Euvre poétique, qui nous épargne toujours l'erreur d'un oubli possible, ou d'un texte égaré, publié ou non... mais la confection en question se propose en tout cas d'être complète, ce qui répond d'avance à une question que l'on pourrait se poser,

1. Louis Aragon, Euvre poétique, I, 1917-1926, Paris, Livre Club Diderot-Messidor, 1989 [1974], p. 10. 
touchant la nature de cette collection. Y figure ce qu'on a retrouvé, que ce qui fut écrit me plaise ou non, aujourd'hui. Voilà, c'est dit.

Qui « préfère » le titre au masculin singulier? L'éditeur? La langue ellemême? Par quel miracle la mort d'un écrivain (que l'édition de ses œuvres permet d'anticiper) suffit-elle à faire passer la collection des œuvres complètes en un œuvre, jugé clos sur lui-même, suffisant? Que cache ce changement de genre? Il y a d'emblée hésitation sur le genre du mot « œuvre ». Or tout au long de cette longue préface qui a pour titre : «Écrit au seuil », l'oscillation entre masculin et féminin semble peu à peu se communiquer à la division entre « le vers » et « la prose », mêlés sous une même couverture, ou aux «diverses alternances sexuelles ${ }^{2}$ » des rimes, puis, quelques pages plus loin, fait retour sous la plume d'Aragon à travers l'ambiguïté du mot « œuvre », qui, mis au féminin, désigne la réunion de toutes les œuvres publiées du vivant de l'auteur et change de genre grammatical à son décès pour désigner... la même chose. Au masculin singulier, « œuvre » s'applique généralement aux productions des peintres ou des graveurs et, lit-on dans Le Grand Robert de la langue française, de manière " plus technique ou affectée » lorsqu'il s'agit d'un écrivain. En la matière, le changement de genre importe moins que le changement de nombre qui a pour effet de fusionner les différentes œuvres auparavant dispersées et de suggérer ainsi une plus grande unité, par conséquent une plus grande exhaustivité. Mais si le choix du masculin est, malgré qu'Aragon en ait, assez peu décisif en ce qui concerne le nombre de textes rassemblés, en revanche, les connotations sexuées qu'il véhicule s'avèrent plus décisives. D'un emploi rare, il se démarque nettement de l'usage au féminin du même mot et évoque toute une série d'associations dont Aragon se saisit lorsque, rappelant qu'avec Breton et ses compagnons surréalistes, il avait déclaré la guerre à ce qui, dans le langage, relève du « sens tout fait», il condamne les avatars de cette révolution littéraire, sa « pratique vulgarisée », non seulement chez « les surréalistes de la génération suivante » mais aussi chez « des littérateurs de niveaux divers, les plus bas » :

Permettez-moi de ne pas salir des lignes de certains exemples. Ceci n'est qu'une simple enseigne, un en-tête plutôt, à ce grand pilori de moi-même, la collection (la décollation) de ce qu'on appelle, au singulier viril, mon Cuvre poétique. Et je n'ai envie que de parler des autres, vous voyez bien ${ }^{3}$.

L'œuvre est ici consignation, obligation faite à l'individu de se rassembler, de se dresser, malgré la menace de castration qui pèse sur celui qui se rigidifie en un corps fait de volumes empilés. D'une certaine manière, les Euvres romanesques croisées de Louis Aragon et Elsa Triolet 
(42 volumes) offraient, par leur alternance, une forme d'hermaphrodisme assumé et épanoui. C'est la fragilité d'un tel équilibre que le jeu autour du masculin singulier, revendiqué et néanmoins redouté, met en évidence au seuil de la dernière grande entreprise éditoriale d'Aragon. Il y a bien un sexe des œuvres - on en discute à perte de vue, comme de celui des anges ou de quelques autres objets tels qu'ils se disent en français.

Si le cas du mot « Mémoires " paraît plus clair et plus tranché, le même jeu entre genres et nombres s'y observe là aussi. Cette fois, l'ambiguitté tient au fait que le genre littéraire des Mémoires (entendus comme " ouvrage faisant le récit des événements dont l'auteur a été le témoin ») s'est construit au XVI ${ }^{\mathrm{e}}$ siècle essentiellement à partir du mot " mémoire », masculin singulier (désignant des écrits destinés à exposer des comptes, une affaire juridique ou une question scientifique), lui-même dérivé «par changement de genre » (Dictionnaire historique de la langue française) au $\mathrm{XII}^{\mathrm{e}}$ de « mémoire », féminin singulier ${ }^{4}$. Certes, le phénomène de dérivation paraît assez facile à reconstituer; reste que la répartition sémantique en fonction du nombre et du genre ne se fait généralement pas aussi aisément : en multipliant les possibilités, elle favorise les risques de confusion. Notamment l'erreur sur le genre, traditionnelle, surtout depuis qu'à partir de la seconde moitié du XIXe , le genre s'est démocratisé, ainsi que Jean-Luc Benoziglio en fait la remarque dans une scène de Cabinet portrait où le narrateur interroge un déménageur sur le comportement de ses clients et se voit répondre :

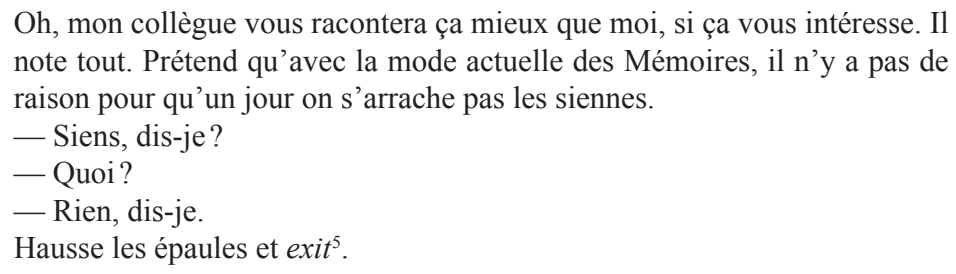

Le phénomène s'est accentué plus récemment avec l'entrée, à partir des années 1980, dans l'ère de la mémoire, plus précisément, l'ère des mémoires collectives et autres lieux de mémoire, puisque l'incroyable extension accordée au féminin pluriel de "mémoire » a souvent eu pour effet d'en faire un substitut de « Mémoires » - il suffit pour s'en convaincre de parcourir la liste de ce qui se publie aujourd'hui sous ce

4. «Des mémoires, avec une minuscule, c'est simplement, à l'origine, le pluriel du substantif masculin, lui-même dérivé de son homonyme féminin. Un mot modeste, un mot de la langue de tous les jours : pour l'avocat, l'apothicaire ou la maîtresse de maison, un mémoire, c'est un écrit dans lequel on consigne tout ce qu'on ne doit pas oublier. » (André Bertière, Le Cardinal de Retz mémorialiste, Paris, Klincksieck, coll. « Bibliothèque française et romaine $», 1977$, p. 12.)

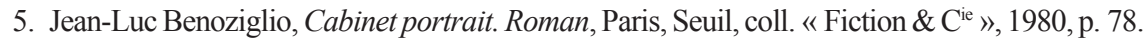


terme : très souvent, il n'est plus question de récits en prose, rétrospectifs et continus dans lesquels un individu raconte les faits dont il fut l'acteur ou le témoin privilégié, mais d'anthologies ou de récits portant sur des pratiques sociales qui ont pour fonction d'entretenir un certain rapport au passé - Mémoires de l'Ardèche paysanne, Mémoires de déportés, Mémoires du désert (sous-titrés : Des Sahéliens se souviennent)... L'erreur (ou la substitution volontaire, je ne sais) est dès lors fréquente : un album de photographies sur le quartier de Lyon consacré à l'industrie de la soie s'intitule (parmi cent autres exemples) Croix-Rousse, mémoires interdites (Éditions EGÉ, 1979). Étonnant phénomène de dilution où le masculin pluriel, appliqué à chaque fois à un texte singulier, s'efface au profit d'un féminin pluriel, étendu à l'ensemble de ce que l'on nomme aujourd'hui de manière très floue « mémoires collectives ».

Mon intention n'est pas de dénoncer une erreur de langue mais de m'en saisir comme d'un prétexte : un prétexte pour interroger l'apparente évidence qui entoure, dans ce cas comme bien souvent en français, l'usage du genre masculin. Les polémiques qui ont entouré les efforts de féminisation du vocabulaire (en 1984, 1986, 1997...) le prouvent amplement ${ }^{6}$ : la grammaire véhicule tout un imaginaire de genre et de sexe d'autant plus prégnant qu'il reste soit implicite (par conséquent rarement discuté) soit (lorsqu'une réforme soulève le lièvre) objet d'un très violent attachement à une norme assimilée de manière confuse au génie de la langue. Ce sont les connotations qui s'attachent à la catégorie littéraire des « Mémoires » que j'aimerais examiner. S'il n'y a aucun sens à s'interroger sur l'application de ce terme au corpus qui nous intéresse au milieu du XVI ${ }^{\mathrm{e}}$ siècle, il est en revanche utile d'interroger les représentations viriles qui s'y attachent depuis lors. Qu'il s'agisse de ses origines nobles (comme les Mémoires d'épée ou les Mémoires d'État), des fonctions qui y prédisposent tout particulièrement (charges militaires, diplomatie, engagements politiques...) ou encore des gestes qui sont attendus de ses auteurs (honneurs obtenus, responsabilités exercées, revendication d'un lignage...), tout semble justifier l'ancrage très largement masculin des Mémoires.

Il n'y a là toutefois rien de naturel, bien au contraire. Nul n'ignore que les Mémoires se sont dès le début écrits au féminin. Marguerite de Valois, Françoise de Motteville, Hortense et Marie Mancini, la duchesse de Montpensier, la marquise de Caylus, Mme de Genlis, Mme Campan, Mme Roland, Georges Sand, la comtesse de Boigne, Marie d'Agoult, Louise Michel sont les plus connues d'une très longue foule et très nombreuse lignée de femmes mémorialistes ${ }^{7}$. Certes, les textes en question ont autrefois

6. Voir Edwige Khaznadar, Le Féminin à la française, Paris, L'Harmattan, 2002; Olivier Houdard et Sylvie Prioul, La Grammaire, c'est pas de la tarte, Paris, Seuil, 2009.

7. Voir notamment Henri Rossi, Mémoires aristocratiques féminins : 1789-1848, Paris, H. Champion, 1998. 
pu faire l'objet de stigmatisations (ce fut le cas par exemple au XIX ${ }^{\mathrm{e}}$ siècle, lorsque l'institution d'une tradition mémoriale jugée typiquement française a conduit à faire le tri parmi les sources disponibles), mais l'essentiel est toutefois que, compte tenu des effets de sélection sociale qu'implique le projet de publier le récit de sa vie, de nombreuses femmes ne se sont pas senties indignes de ce genre. Pourquoi ont-elles dès lors été si rares à livrer un récit «égohistorique » (ainsi peut-on désigner la représentation que l'on livre de soi-même dans sa condition historique) précisément à partir du moment où elles ont conquis toute une série de droits et bénéficié d'une égalité, il est vrai encore bien incomplète, mais incomparable avec leur situation passée? On supposera peut-être que le statut de reine ou d'aristocrate, le fait de tenir salon ou même la participation à des événements révolutionnaires ont pu favoriser l'émergence de figures mémoriales féminines là où l'accès au droit de vote ou les efforts pour faire respecter la parité ont paradoxalement détourné les femmes d'écrire l'histoire - mais cela paraîtra bien simpliste. Une autre réponse se présente immédiatement à l'esprit : une sorte d'évolution naturelle des genres expliquerait ce lent passage des récits de «Vies majuscules 》 à des formes de récits plus intimes (journaux, correspondances, autobiographies...), plus propices à l'expression de l'identité féminine. L'idée repose sur un syllogisme dont la prémisse majeure est que les genres à la première personne rendent de plus en plus directement compte de l'individu moderne dans son intimité et la prémisse mineure est que les femmes sont portées à l'expression subjective de soi, la conjonction des deux expliquant la proportion importante d'autobiographies féminines. Le problème est que cette prémisse majeure se voit infirmée par les faits - on publie au $\mathrm{Xx}^{\mathrm{e}}$ siècle tout autant de Mémoires que par le passé - et que la prémisse mineure repose sur un postulat tout à fait contestable. J'aimerais ici me demander si le fait de se limiter - ou de se voir limiter - aux modèles autobiographiques au détriment du pôle égohistoriques (Souvenirs, Mémoires, témoignages historiques...) n'a pas amoindri la place que les femmes sont en droit d'occuper dans le champ des récits de soi et ne les a pas confinées à une sorte de gynécée des écrits à la première personne.

\section{Vies de femme majuscules}

Pour commencer, un très rapide bilan de la production mémoriale féminine au $\mathrm{xx}^{\mathrm{e}}$ siècle. Et un test avant d'en venir à l'examen des bibliographies disponibles: Beauvoir exceptée, combien de célèbres femmes mémorialistes après George Sand et Louise Michel pouvons-nous citer, si nous nous donnons une définition relativement stricte du genre? Pour ma part, je l'avoue, les candidates ne sont pas nombreuses. On me répondra qu'en me limitant à un modèle traditionnel des Mémoires (dominé par le canon des grands Mémoires d'État), je fausse nécessairement le jeu et exclus toute une quantité d'écrivaines ayant à l'inverse visé le renouvellement du 
modèle mémorial et son hybridation avec toutes sortes de formes connexes, plus propices à l'introspection. Mais ce serait, là encore, ignorer ce qui me paraît être l'enjeu principal d'une lecture genrée de l'histoire des Vies majuscules, à savoir se demander pour quelle raison, alors qu'elles n'hésitaient pas jusqu'au XIX ${ }^{\mathrm{e}}$ siècle à écrire l'histoire de leur temps, les femmes ne revendiqueraient plus - ou ne se verraient plus reconnaître - un tel droit (sous sa variante mémoriale) de nos jours.

En débutant en 1996 mes recherches sur les Mémoires au xx siècle, je me suis engagé dans un long dépouillement bibliographique et, afin de faire varier les échelles, me suis efforcé d'établir deux corpus. Le premier regroupait les Mémoires au sens le plus large de ce terme : l'unique critère retenu était celui de l'inscription du terme générique dans le titre - je ne prêtais, autrement dit, aucune attention aux thèmes développés ou aux formes empruntées, mais uniquement au fait que l'auteur (ou l'éditeur) des volumes avait choisi de recourir à ce nom de genre particulier, quelle que soit la raison de son choix. Ce corpus « extensif » où se croisaient des journaux, des entretiens, des récits de vie, des autobiographies, des romans ou même des recueils de poésie et des ouvrages érotiques ou humoristiques comportait un nombre relativement important d'écrivaines. Le second corpus, « limitatif », concernait les Mémoires envisagés non plus en extension mais en compréhension, c'est-à-dire conformes au canon hérité des siècles passés et pouvant être très simplement définis comme le récit rétrospectif et continu qu'un individu fait des événements historiques auxquels il a participé ou dont il a été témoin. Ce corpus se composait de quelque cinq cent cinquante textes ${ }^{8}$. Il résulte du croisement des deux corpus une sélection de vingt-six auteures de Mémoires au sens restreint du terme ${ }^{9}$, parmi lesquelles notamment Juliette Adam, Dominique Desanti, Annie Kriegel, Lise London, Jeannette Thorez-Vermeersch, et Louise Weiss. Vingt-huit sur cinq cents, le rapport est, on le voit, nettement défavorable aux femmes. En revanche, celles-ci s'avèrent beaucoup plus nombreuses lorsque l'on puise dans le corpus extensif, puisqu'y abondent les Mémoires d'aristocrates (comme Mémoires : au temps des équipages, d'Élisabeth de Gramont) - que j'ai classés dans les Souvenirs en raison de leur caractère totalement anecdotique, sans plus aucune véritable prise sur l'Histoire -, les

8. J'insiste sur le fait qu'il ne s'agit pas, en dégageant un corpus fermé, de délimiter une identité générique immuable. Le modèle des Mémoires d'État, si important soit-il en raison de la place qu'occupe cette tradition littéraire et historiographique en France, ne peut prétendre à aucun monopole générique.

9. Juliette Adam, Victorine B., Simone de Beauvoir, Suzanne Bidault, Suzanne Blum, Jeanne Bohec, Marie Boudier-Salter, Janine Bouissounouse, Dominique Desanti, Anne-Marie Dupuy, Odette Fabius, Marie-Madeleine Fourcade, Brigitte Friang, Annie Kriegel, Lise London, Simone Martin-Chauffier, Suzanne Massu, Lucie Mazauric, Élizabeth de Miribel, Henriette Nizan, Cécile Ouzoulias-Roumagon, Amédée Ozenfant, Jeannette Thorez-Vermeersch, Anna Trahin, Louise Weiss. 
témoignages de vedettes (Toute ma vie, de Mistinguett, Une vie de toutes les couleurs de Joséphine Baker, La Nostalgie n'est plus ce qu'elle était, de Simone Signoret...), les récits hybrides (comme Une enfance stalinienne, très beau texte où Lily Marcou livre un témoignage sur l'habitus communiste après la Seconde Guerre mondiale, ou les Mémoires précoces suivis des Contre-Mémoires de Françoise d'Eaubonne, qui invente une forme narrative où les fils temporels se croisent et se court-circuitent de façon révélatrice ${ }^{10}$ ), enfin toutes sortes de parcours professionnels et de récits de vies ordinaires.

Un point me frappe : c'est que parmi les femmes qui ont choisi d'écrire des Vies majuscules traditionnelles, en dehors de quelques cas particuliers où les mémorialistes font preuve d'une grande inventivité formelle (telle Louise Weiss), le plus souvent rien de particulier ne distingue leur récit de celui des hommes à la même époque. Bien entendu, il est facile d'identifier certains points de divergence sexuée : on peut ainsi juger que là où les hommes reconstituent leur parcours de vie sur fond d'événements politiques ou historiques, les femmes semblent raconter plus volontiers l'histoire d'un couple, à commencer d'ailleurs par l'auteure de La Force de l'âge et La Force des choses: Suzanne Bidault, Dominique Desanti, Suzanne Massu, Henriette Nizan, ou Jeannette Thorez-Vermeersch évoquent, comme Clara Malraux dans Le Bruit de nos pas, une histoire vécue pour une large partie à deux. Reste que si une telle divergence est avérée, elle devrait, en toute logique, être tout aussi révélatrice de la " nature » supposée des mémorialistes masculins; or, nous sommes bien entendu plus réticents à tirer une telle conclusion - tout du moins plus enclins à juger qu'elle relève d'un lieu commun qu'il serait peu rigoureux d'explorer. La question est en quelque sorte moins de savoir s'il existe des différences notables entre les Mémoires de femmes et leurs équivalents masculins, que de s'interroger sur les présupposés d'une telle approche. Car le point essentiel me paraît être que nous aimerions qu'il en aille de la sorte : nous aimerions (quels que soient les mobiles d'un tel désir) qu'il soit possible de produire des divergences significatives - autrement dit montrer que parce qu'ils ont été écrits par des femmes, les textes considérés peuvent être considérés comme " féminins », alors qu'il y a là une déduction hâtive, et qui n'est que le pendant du présupposé inverse selon lequel parce que les Mémoires sont souvent signés par des hommes, le genre serait en soi « masculin ».

10. Voir Françoise d'Eaubonne, Chienne de jeunesse. Mémoires précoces 1 et Les Monstres de l'été. Mémoires précoces 2, Paris, Julliard, 1965-1966, puis L'Indicateur du réseau: contre-mémoires, Paris, Encre, 1980. L'ensemble des Mémoires de Françoise d'Eaubonne a été republié sous le titre : Mémoires irréductibles : de l'entre-deux-guerres à l'an 2000 chez Dagorno en 2001. 


\section{La femme « en général »...}

La question est complexe, car s'y mélangent à la fois les préjugés les plus misogynes et les bonnes intentions féministes les plus louables. En effet, on peut, par souci de valoriser un corpus de récits de soi féminins, reprocher aux auteures de Vies majuscules de s'en tenir à un témoignage historique, comme si un récit principalement exogène avait par définition moins de valeur que les récits plus personnels publiés au cours de ce même siècle par quantité d'autobiographes, de diaristes ou d'épistolières. Outre le fait qu'il me semble totalement faux de privilégier par principe l'intime sur l'historique, un tel jugement me paraît discutable pour des raisons d'ordre théorique et politique. Attendre des femmes que leur production reflète, d'une manière ou d'une autre, un trait quelconque de leur identité de genre supposée (une tendance introspective, une attention particulière à la vie émotionnelle et affective, ou encore, comme je viens de le faire moi-même, un plus grand soucis des relations de couple) revient à hypostasier une nature féminine : Clara Malraux a beau parler plus souvent d'André que d'elle-même dans Le Bruit de nos pas (où celui-ci n'est absent que dans le premier et le dernier des six tomes), alors que l'auteur du Miroir des limbes ne mentionne même pas le nom de sa première épouse, on ne saurait en faire un argument pour divaguer sur les différences entre hommes et femmes. Spontanément, nous aimerions pouvoir revaloriser les Vies majuscules de femmes en mettant au jour des traits singuliers de leur "sensibilité ». Mais une telle stratégie n'est jamais que l'envers des préjugés dont la littérature féminine fait souvent l'objet. Bien sûr, le temps n'est plus où l'on pensait que lorsqu'une « femme se raconte », on lui reproche aussitôt « de n'être plus femme $\gg{ }^{11}$, ainsi que le notait Marguerite Yourcenar; aujourd'hui, on attendrait plutôt, à l'inverse, d'une femme qui écrit qu'elle se raconte automatiquement dans son intimité. J'en trouve un exemple frappant dans le bilan sur la place des femmes de lettres dans les histoires littéraires que Michèle Touret établit en tant que directrice de l'Histoire de la littérature française $d u x X^{e}$ siècle publiée en deux volumes, en 2000 et en 2008, aux Presses universitaires de Rennes pour le numéro 7 de la revue en ligne Fabula-LHT, dirigé par Audrey Lasserre. Michèle Touret note, en effet, l' « importance du journal, de l'écrit intime dans la production féminine », qu'elle oppose aussitôt aux « Mémoires au sens ancien du terme dans la mesure où ceux-ci concernent la place de l'individu dans l'histoire générale de son temps et sont généralement le fait des hommes ${ }^{12}{ }^{2}$, comme si un état de fait contemporain (le corpus réduit de Mémoires publiés par des

11. Marguerite Yourcenar, Carnets de notes de Mémoires d'Hadrien, dans Euvres romanesques, Paris, Gallimard, coll. «Bibliothèque de la Pléiade », 1982, p. 526.

12. Michèle Touret, « Où sont-elles? Que font-elles? La place des femmes dans l'histoire littéraire. Un point de vue de vingtiémiste », Fabula-LHT [En ligne], n 7 : Audrey Lasserre (dir.), « Y a-t-il une histoire littéraire des femmes? », septembre 2010 (http://www.fabula. org/lht/7/dossier/185-7touret). 
femmes), joint au présupposé communément partagé que la tendance « naturelle » des femmes serait de se confier, suffisait à gommer qu' au cours des siècles classiques et jusqu'au XIX ${ }^{\mathrm{e}}$ siècle, des reines, des combattantes, des témoins proches du pouvoir, ou des salonnières écrivirent à la première personne l'histoire de leur temps. Tout se passe comme si en cherchant à sauver les femmes de l'oubli en valorisant leur propension à l'expression de l'intime, on leur interdisait un droit à mon sens plus fondamental à l'expression du mémorable.

Car les femmes ont-elles à gagner à se voir ainsi assigner une caractéristique dont la valorisation entraîne incontinent une réduction de leur sphère d'exercice? Leur attribuer une compétence autobiographie particulière revient, me semble-t-il, à entériner cette remarque de Julien Gracq qui condense tous les préjugés dont les Mémoires féminins peuvent faire l'objet (je précise que je me suis interdit de puiser mon exemple dans Le Journal inutile de Paul Morand - le résultat aurait été si caricatural qu'il aurait perdu toute valeur) :

Les neuf dixièmes de notre temps vécu, de ce temps dont rien après tout n'est inintéressant pour la littérature, se déroulent dans un monde sans passé et sans avenir, dans le monde de ce qu'Éluard a nommé la Vie immédiate, monde où l'histoire mord à peine, où le souci de l'action et de l'engagement n'a pas de prise. Le monde énorme du rêve, du rêve endormi et du rêve éveillé, échappe à [la littérature contemporaine]. Celui des paysages. Celui, tout de même immense, des religions. Celui de l'amour, qui refuse les servitudes de temps et de lieu. L'univers féminin à peu près tout entier, c'est-à-dire la moitié du monde de la conscience : la femme en général ne se sent guère dans l'histoire : chercher à l'introduire dans une œuvre aussi complètement féminine que celle de Colette, c'est chercher une place libre dans un œuf lisse et fermé ${ }^{13}$.

Déduire des textes de Colette une vérité sur «la femme en général » ainsi que l'écrit Gracq : qui se permettrait aujourd'hui l'équivalent à partir d'André Malraux (l'exercice consisterait à divaguer sur la nature profondément virile et exogène de ses romans au personnages quasi exclusivement masculins) ou de Marcel Proust (avec qui le processus de généralisation se révèlerait particulièrement délicat)? Toute spéculation de ce type s'accompagne d'un coût intellectuel et politique : il est indéniable que les femmes se sont au $\mathrm{Xx}^{\mathrm{e}}$ siècle pleinement approprié le modèle autobiographique; quant à savoir ce qu'il faut en déduire sur les liens véritables des femmes à l'autobiographie ou aux Mémoires, on risque toujours de glisser d'un constat s'appuyant sur des déterminismes subis à une hypothèse sur des propensions jugées «naturelles ». En la matière, les bonnes intentions militantes risquent d'être tout aussi dangereuses

13. Julien Gracq, « Pourquoi la littérature respire mal », Préférences, dans Euvres complètes, t. I, éd. Bernhild Boie, Paris, Gallimard, coll. «Bibliothèque de la Pléiade », 1989, p. 878. 
que le jugement à l'emporte-pièce de Julien Gracq. La position la plus prudente et la plus rigoureuse me paraît être (je m'autorise ici des analyses de Michèle Le Doeuff sur ce qu'elle appelle Le Sexe du savoir) de ne rien supposer, ou plutôt de supposer que les femmes jouissent en droit et par principe d'une égale disposition aux Mémoires - ce qui n'implique, bien entendu, pas que les conditions sociales, intellectuelles ou éditoriales qui leur sont faites leur permettent d'y prétendre réellement.

C'est pourquoi je préfère m'en tenir au fait que dans le cas des textes où des femmes ont choisi de raconter leur engagement dans la résistance, leurs luttes politiques, leur parcours intellectuel, ou leur traversée d'un demisiècle (ainsi que Catherine Clément vient de le faire en publiant un texte sobrement intitulé : Mémoires, paru chez Stock en 2009), leur identité de genre n'a pas influencé de manière décisive leur usage du modèle mémorial. C'est la raison pour laquelle la percée des femmes dans le domaine autobiographique au $\mathrm{XX}^{\mathrm{e}}$ siècle, tout à fait essentielle pour le développement de ce champ narratif ${ }^{14}$, me paraît cependant moins importante que sa contrepartie, à savoir leur dépossession de l'exercice d'un droit à écrire l'Histoire - sous cette forme particulière qu'est le genre des Vies majuscules tout du moins, puisqu'à partir des années 1970 est apparue une histoire des femmes, représentée en France par Michelle Perrot, Christine Bard ou Françoise Thébaud $^{15}$. Dans les faits, il y a donc bien un sexe des Mémoires, mais qui varie selon les époques et se confond avec celui de ses contributeurs/trices, en sorte que le nombre plutôt réduit de mémorialistes femmes au $\mathrm{Xx}^{\mathrm{e}}$ siècle soulève une question littéraire et politique essentielle - pour autant que l'on admet que les Vies majuscules exercent un effet sur la représentation que les membres d'une communauté se font de leur passé récent.

\section{« C'est l'homme qu'il faut chercher ici, et si on ne le trouve pas, il n'y a rien. " (Albert Sorel)}

Afin d'interroger cette distribution d'un genre littéraire en fonction des genres sexués, forme particulière et fluctuante au cours des siècles de ce qu'Erwin Goffman nomme « l'arrangement des sexes », j'aimerais saisir cette question à son point d'articulation historique, c'est-à-dire au moment même où les femmes apparaissent, pour la dernière fois, comme

14. Sur ce point, voir l'article d'Éliane Lecarme-Tabone, « L'autobiographie des femmes », Fabula-LHT [En ligne], n ${ }^{\circ} 7$ : Audrey Lasserre (dir.), «Y a-t-il une histoire littéraire des femmes? », septembre 2010 (http://www.fabula.org/lht/7/dossier/168-7lecarme-tabone).

15. Voir par exemple Johanna Alberti, Gender and the Historian, Harlow, Pearson Education, 2002 ; Mary Spongberg, Writing Women's History since the Renaissance, New York, Palgrave Macmillan, 2002; Nicole Pellegrin, Histoires d'historiennes, Saint-Étienne, Publications de l'Université de Saint-Étienne, 2006; Sylvie Steinberg et Jean-Claude Arnould (dir.), Les Femmes et l'écriture de l'histoire, 1400-1800, Mont-Saint-Aignan, Publications des universités de Rouen et du Havre, 2008. 
des représentantes autorisées et puissantes (par leur nombre tout du moins) du genre mémorial, et se voient néanmoins frappées d'illégitimité, comme chassées de ce domaine d'exercice au profit de la sphère intime. Exemplaire à ce titre me semble la synthèse qu'Albert Sorel, professeur d'histoire diplomatique à l'École libre des sciences politiques et successeur de Taine à l'Académie française, publia dans Minerva en 1903. Tout l'effort de légitimation des Vies majuscules par les historiens romantiques trouve son aboutissement dans ce texte, où ce genre se voit confirmer son statut d'auxiliaire privilégié de l'histoire - cinq ans après que Langlois et Seignobos, bien plus influents que Sorel, lui ont refusé de telles prérogatives dans leur Introduction aux études historiques. Le texte de Sorel n'est donc pas en phase avec les évolutions que connaît alors la discipline sous l'influence des historiens « méthodistes », fort méfiants à l'égard de témoignages perçus comme bien trop apologétiques, mais s'avère néanmoins intéressant par les préjugés qu'il condense et auxquels il confère une plus grande densité que partout ailleurs.

$\mathrm{Au}$ cours de son examen des différentes formes prises par les Vies majuscules, Sorel aborde ainsi le cas de notre corpus :

Je devrais, pour être complet, parler des Mémoires de femmes; mais il faudrait tout recommencer en rhabillant tout à leur taille. Aussi bien le lecteur trouvera-t-il aisément la transposition. J'avoue que je n'ai pas beaucoup plus de goût pour les mémoires politiques des femmes que pour les femmes politiques elles-mêmes. Celles qui ont eu du génie politique et qui sont de véritables monstres, en leur sexe, ont écrit, comme elles agissaient, en hommes. Celles qui ont vécu et écrit en femmes d'imagination et de cœur, qu'elles étaient, comme Madame de Staël, en ses Années d'exil, ont laissé à la postérité le beau rêve d'une politique illusoire: témoins éloquents, touchants, pathétiques des généreuses utopies de leur temps. Il y a beaucoup de politique dans les Mémoires de Madame Roland, mais que de préventions féminines, de partialités en cette politique-là, et comme il faut y faire la part même de la répulsion physique, pour Danton, par exemple, ou, ailleurs, de la séduction toute personnelle, de la voix, du regard! Confessions ici; commérages chez la duchesse d'Abrantès, confidences chez l'aimable chanoinesse de Chastenay. La plus politique, au fond, est Madame de Rémusat, qui a dû brûler ses mémoires en 1814, afin, comme son ami Talleyrand, qui peut-être lui en a soufflé plus d'un passage, de les remettre au goût de la société nouvelle appelée à les connaître.

Ce qu'il faut lire toujours, en ces Mémoires de femmes, et relire, et méditer et savourer, c'est la partie vraiment féminine et naturelle, la partie intéressante et intéressée, les affaires de cœur, d'amour, de jalousie. Les femmes ont la vision, le trait, le mot, elles égratignent, mais le sang paraît et c'est la vie. Dans la politique, quand elles en parlent, l'œuvre est presque toujours artificielle, de reflet. Ce n'est plus le miroir où elles se regardent, c'est la miniature où elles regardent leur amant. C'est l'homme qu'il faut chercher ici, et si on ne le trouve pas, il n'y a rien. Elles sont trop préoccupées, trop obsédées de se créer un personnage, de se faire un salon dans l'histoire, un salon où l'on cause, et où l'on cabale davantage, et d'en faire le rouage principal de la machine. Il faut s'attacher surtout à ce 


\begin{abstract}
qu'elles ont observé sans le vouloir, cueilli comme au hasard, au passage, à ce qu'elles rapportent sans y donner d'importance : elles n'y ont pas retouché, et il y a des chances pour que ce soit vrai. Leurs Mémoires sont semés de ces traits-là; il y en a de singulièrement suggestifs dans les petits cahiers de Madame de Coigny. Pour leurs impressions sur les hommes, sur les événements, plus importantes, chez elles, que les faits mêmes, se demander toujours de quel côté elles regardaient le jour dont elles parlent, et de quel côté elles regardaient encore le jour où elles ont écrit ${ }^{16}$.
\end{abstract}

On ne peut qu'être frappé par les effets ravageurs de la logique essentialiste. Les mémorialistes se trouvent ici condamnées à une sorte de doublebind: lorsqu'elles s'occupent des affaires de leur temps, les femmes se muent en hommes et inspirent dès lors une sorte de répulsion qu'Albert Sorel n'éprouve même pas le besoin de justifier; mais si elles se limitent à l'inverse à la sphère de la vie privée, leur témoignage n'a plus qu'un intérêt anecdotique, récréatif en quelque sorte. Un adage, véritable loi garantissant le bon usage des Mémoires, résume l'enjeu : «C'est l'homme qu'il faut chercher ici, et si on ne le trouve pas, il n'y a rien. »

L'analyse de l'historien s'avère toutefois moins intéressante que la réponse indirecte que lui apporta Marcel Proust, son ancien élève à l'École libre des sciences politiques, tout d'abord dans « Journées de lecture ", puis dans « À une snob» (chapitre des Plaisirs et les jours), enfin dans $\grave{A}$ la recherche $d u$ temps perdu, où Mme de Villeparisis incarne la mémorialiste que le jeune écrivain avait décrite dans "Journées de lecture » comme « cette sorte de personnes, souvent très bien nées, mais, on ne sait pourquoi, peu recherchées et que les femmes à la mode désignent volontiers par l'appellation de "chameaux"17 ». La sinueuse réflexion du narrateur de ce texte part des Mémoires de la comtesse de Boigne : le chameau y devient un puissant stratège capable de regagner grâce aux Mémoires, et aux déformations qu'autorise le fait de citer tel nom, de glisser telle anecdote, de se placer au centre des attentions de tel homme célèbre, ce que sa vie mondaine n'avait pas réussi à lui apporter de succès. Habile tactique, qui font des Vies majuscules un champ de bataille où le snobisme se substitue à la gloire militaire ou politique et où les femmes, parce qu'elles dirigent les salons, véritables centres vitaux de la vie mondaine, éclipsent les généraux et les diplomates. L'analyse que fait Proust de ce phénomène s'avère étonnante. Prenant acte de la présence de plus en plus massive au $\mathrm{XIX}^{\mathrm{e}}$ siècle des femmes dans le champ mémorial, celui-ci redessine l'histoire récente du genre; là où son ancien professeur tentait de limiter les dégâts au regard de la tradition des Mémoires d'État, il entérine la réduction que dénonçait Albert Sorel tout en y voyant le charme véritable de ces récits :

16. Albert Sorel, « Histoire et Mémoires », Minerva, n 22, 15 janvier 1903, p. 175-176. 17. Marcel Proust, «Journées de lecture », Contre Sainte-Beuve, précédé de Pastiches et mélanges et suivi de Essais et articles, éd. Pierre Clarac et Yves Sandre, Paris, Gallimard, coll. «Bibliothèque de la Pléiade », 1971, p. 926. 
Les Mémoires de la fin du XVIII ${ }^{\mathrm{e}}$ siècle et du commencement du XIX ${ }^{\mathrm{e}}$, comme ceux de la comtesse de Boigne, ont ceci d'émouvant qu'ils donnent à l'époque contemporaine, à nos jours vécus sans beauté, une perspective assez noble et assez mélancolique, en faisant d'eux comme le premier plan de l'Histoire ${ }^{18}$.

La chaîne des liens que tissent les souvenirs et les récits anecdotiques donne l'impression d'une proximité avec le passé envisagé à travers le prisme des fréquentations, mais elle ne livre accès qu'à une histoire transformée en décor, simple fond sur lequel l'époque contemporaine se détache. Le passé historique se voit chez ces mémorialistes tout entier contaminé par les codes de la mondanité : les femmes deviennent les détentrices d'une nouvelle sensibilité, la frivolité, envisagée comme une forme de rapport au passé que Proust n'hésite pas à comparer aux extraordinaires découvertes des archéologues et des archivistes, pour qui « rien n'est oublié »; le récit détaillée d'une mémorialiste prouve ainsi, mieux que la découverte d'un monument, que «la plus chétive circonstance de la vie, la plus éloignée de nous, est allée marquer son sillon dans les immenses catacombes du passé où l'humanité raconte sa vie heure par heure ${ }^{19}{ }^{\prime}$. L'intérêt des Vies majuscules est dès lors d'exhiber des personnages comme « ces personnages en cire et grandeur nature qui, au premier plan des panoramas, foulant aux pieds de l'herbe vraie et levant en l'air une canne achetée chez le marchand, semblent encore appartenir à la foule qui les regarde, et nous conduisent peu à peu à la toile peinte du fond, à qui ils donnent, grâce à des transitions habilement ménagées, l'apparence du relief de la réalité et de la vie ${ }^{20} »$. Voici l'histoire devenue une toile de fond, n'ayant plus de réalité qu'à travers l'effet d'optique qu'autorisent la transmission des noms et la continuité des grandes familles aristocratiques. La mondanité est une dimension inhérente au genre lui-même; mais là où Saint-Simon peignait un microcosme qui détenait le pouvoir, Mme de Boigne décrit un univers désormais privé de ses prérogatives sociales et politiques les plus tangibles - et qui à l'époque de Proust ne dispose plus pour l'essentiel que d'un capital symbolique.

L'analogie de Proust entre les Mémoires et les panoramas révèle la conséquence d'une telle évolution, à savoir la mise à plat de la profondeur historique dont jouissaient les textes mémoriaux. Cette mutation essentielle du genre, ainsi transformé en une simple chronique de la vie mondaine, est à ses yeux étroitement liée à sa conquête par des femmes et son invasion par la sociabilité salonnière qu'elles dominent. Processus ambigu, où Mme de Boigne et ses semblables acquièrent une forme de reconnaissance mais cela au moment où le genre arrive à épuisement : $\grave{A}$ la recherche du temps perdu va recueillir l'héritage de la longue tradition mémoriale, redéployée

18. Ibid., p. 531.

19. Ibid., p. 925.

20. Ibid., p. 531-532. 
dans l'univers fictionnel, à travers les rêveries du narrateur sur l'essence d'une caste qu'il pare de tous les prestiges, son entrée dans un monde dont les codes obéissent à un constant souci de distinction, son intérêt pour l'onomastique ou les généalogies dont Charlus se fait le sourcilleux garant, puis sa découverte de l'inéluctable déclin de l'aristocratie dont la mécanique ou les préjugés se communiquent à d'autres milieux - le bouleversement final de « la charte des situations » que décrit La Recherche fait bien de Proust à la fois le successeur de Saint-Simon et le liquidateur de cet imaginaire mémorial.

La III République est précisément le moment où le genre des Vies majuscules connaît l'un de ses plus profonds moments de déclin. Plusieurs facteurs sont à considérer. Premièrement, le reflux de la violence révolutionnaire qui avait longtemps divisé la communauté nationale jusqu'à ce dernier sursaut de guerre civile que fut la Commune, et la régulation du pouvoir politique exercé par une classe politique n'ayant plus à offrir de destins exceptionnels (un homme comme Clemenceau n'écrira ses Mémoires qu'en réponse aux polémiques lancées par Foch et sans donner à son récit la hauteur attendue). Deuxièmement, l'appauvrissement du modèle que montrent les témoignages de grands chefs militaires, ou de diplomates publiés après la Grande Guerre, au point de vue étroitement militaire ou politique ne permettant pas de rendre compte de l'expérience vécue par les soldats français - c'est précisément à cette époque que les formes de souvenirs collectifs prennent l'ampleur que nous leur connaissons aujourd'hui : constructions de monuments, développement des pratiques commémoratives, publication en masse de témoignages ou de correspondances d'individus ordinaires... Troisièmement, l'expulsion de ce genre vieux de plus de cinq siècles de ses deux domaines d'exercice privilégiés, l'histoire ainsi que nous l'avons vu, et la littérature, où l'autobiographie est progressivement devenue depuis la fin du XIX ${ }^{\mathrm{e}}$ siècle un modèle de récit de soi plus légitime. La synthèse d'Albert Sorel obéit donc manifestement à une fonction prophylactique, mais en vain : le transfert des champs de bataille aux salons mondains est déjà largement amorcé, ainsi que le montre Proust, et il ne sert à rien de prétendre redonner aux Mémoires une valeur historiographique qu'ils ont déjà perdue, en cherchant à les expurger de leur composante féminine.

Dans ce contexte très particulier de déclin, les deux interventions de Sorel et de Proust ont en commun de faire du sexe des mémorialistes un facteur clé de détermination et d'évaluation des Vies majuscules. Qu'elle vise à dénoncer le rôle joué par les femmes ou au contraire à l'illustrer, cette assignation identitaire s'avère dommageable pour celles-ci. Proust a beau priser la littérature de salon qui connaît à l'époque une formidable extension, sa lecture de la comtesse de Boigne lui livre un contre-modèle que Mme de Villeparisis incarnera au cœur même d' $̇$ la recherche du temps perdu - la lecture dans Le Temps retrouvé des Goncourt, dont la littérature 
est l'émanation de cet univers des salons et des valeurs qui le structurent, achèvera le processus par lequel Proust transmue un modèle mémorial réduit à une chronique mondaine en roman de la mémoire involontaire. Là me semble l'essentiel, à savoir le caractère ambigu de toute spéculation sur le sexe des Mémoires, quand bien même il s'agit d'y défendre le rôle joué par les femmes. Qu'ils visent à les stigmatiser ou à les valoriser, les discours qui portent sur la contribution du deuxième sexe sont rarement sans effets négatifs pour elles. Il y a là une sorte d'impasse, qu'il est fort délicat d'éviter. Depuis plusieurs années sont conduits des travaux particulièrement utiles sur les mémorialistes femmes des siècles classiques : si ces publications contribuent à faire connaître des œuvres quelque peu négligées et à soulever d'intéressantes questions sur leur place dans l'histoire du genre, peut-être l'avenir nous montrera-t-il qu'une telle approche militante a pour effet involontaire de fausser la perspective et de nous conduire à lire leurs textes plus pour ce que ces derniers nous révèleraient des femmes (ou de ce que nous pensons des femmes, à l'avenir certainement aussi caricatural qu'à la Belle Époque) que pour ce qu'ils nous apprennent de l'Histoire.

Peut-être l'unique manière de se prémunir des dangers dont il vient d'être question est-elle avant tout de reconnaître nos limites en la matière. J'aimerais donc conclure en revenant à l'épineuse question de la singularité des Mémoires féminins, mais en m'impliquant dans cette question, au lieu de prétendre pouvoir y répondre dans l'abstrait. Même si je considère qu'il s'agit d'une fausse question, ou plutôt d'une sorte de piège, je ne reconnais pas moins le caractère déterminant du sexe (biologique et/ou social) au regard de l'histoire du genre mémorial. Elle a pour principal intérêt de nous contraindre à débusquer dans chacune de nos analyses une part d'aveuglement. Car en débutant cet article par quelques références bibliographiques, je me suis retranché derrière des données qui se voulaient objectives. Vaine illusion : à reparcourir aujourd'hui ces corpus constitués lors du travail de thèse, je suis frappé des a priori sexistes qui s'y cachent. Pourquoi en avoir exclu Lucie Aubrac, Dominique Auclères, Françoise d'Eaubonne, Élisabeth de Gramont, Françoise Verny, ou d'autres? Dans chacun des cas, des raisons formelles m'ont conduit à distinguer leur textes du modèle canonique : Françoise d'Eaubonne par exemple en raison du caractère plus expérimental de son récit, Élisabeth de Gramont par sa trop grande proximité avec les Souvenirs mondains, Françoise Verny parce que Le Plus beau métier du monde emprunte le modèle du parcours professionnel... Reste que non seulement le parcours du Tableau de la littérature autobiographique féminine de langue française publiée en France de 1944 à 1994 par Élisabeth Sire montre qu'une partie assez importante des textes publiés à cette époque sont intitulés « Mémoires » par leurs auteures, mais que, de plus, dans différents cas, la part de ce que j'ai considéré comme préoccupations secondaires par rapport au récit mémorial traditionnel 
fut plus importante dans le cas des femmes que dans celui des hommes. En sorte que si, après George Sand, les femmes ont bien massivement délaissé le domaine des Vies majuscules, ce constat ne doit être fait qu'avec prudence et pondéré par un facteur stratégique, à savoir notre indéracinable propension à confiner les femmes, malgré qu'elles en aient, à un domaine d'expression. $\mathrm{Au} \mathrm{Xx}^{\mathrm{e}}$ siècle, les femmes ont contribué à écrire l'Histoire, non pas nécessairement sous une forme qui n'appartiendraient qu'à elle, mais plus simplement en dépit de nos grilles de lecture, qui nous cachent le plus souvent leur contribution.

Jean-Louis Jeannelle

Université Paris-Sorbonne-IUF 